\title{
Clinical and pathologic features for predicting malignancy in thyroid follicular neoplasms
}

\author{
Kwangsoon Kim ${ }^{1}$, Chan Kwon Jung ${ }^{2}$, Dong-Jun Lim ${ }^{3}$, Ja Seong Bae ${ }^{1}$, Jeong Soo Kim ${ }^{1}$ \\ ${ }^{1}$ Department of Surgery, College of Medicine, the Catholic University of Korea, Seoul, Korea; ${ }^{2}$ Department of Hospital Pathology, College of \\ Medicine, the Catholic University of Korea, Seoul, Korea; ${ }^{3}$ Division of Endocrinology and Metabolism, Department of Internal Medicine, College \\ of Medicine, the Catholic University of Korea, Seoul, Korea \\ Contributions: (I) Conception and design: K Kim, JS Bae; (II) Administrative support: CK Jung, DJ Lim; (III) Provision of study materials or patients: \\ K Kim, JS Bae; (IV) Collection and assembly of data: K Kim, JS Bae; (V) Data analysis and interpretation: K Kim, CK Jung, DJ Lim; (VI) Manuscript \\ writing: All authors; (VII) Final approval of manuscript: All authors. \\ Correspondence to: Ja Seong Bae, MD, PhD. Professor, Department of Surgery, College of Medicine, the Catholic University of Korea, 06591, Seoul, \\ Korea. Email: Jaseong@gmail.com.
}

Background: The cytologic findings of follicular neoplasm do not distinguish between benign follicular
adenoma and follicular thyroid carcinoma (FTC). The objective of this retrospective study was to identify
clinical and cytologic/pathologic features to predict malignancy in patients preoperatively diagnosed with
follicular neoplasms.
Methods: In total, 416 patients with follicular neoplasms who underwent thyroidectomy were reviewed at
Seoul St. Mary's Hospital (Seoul, Korea) from January 2010 to June 2018 . Clinicopathological features were
analyzed retrospectively by complete medical chart review and pathologic slide review.
Results: Thyroid malignancy/noninvasive follicular thyroid neoplasm with papillary-like nuclear features
(NIFTP) was diagnosed in 209 patients ( $50.2 \%$ ). In total, 59 patients (14.2\%) were diagnosed with FTC, 55
patients (13.3\%) were diagnosed with follicular variant papillary thyroid carcinoma (fvPTC). The number
of patients with PTC-related nuclear changes was higher in the malignancy/NIFTP group than in the
benign group (16.3\% vs. $1.9 \%, \mathrm{P}<0.001$ ). Multivariate analysis indicated that the significant risk factors for
the diagnosis of malignancy/NIFTP include cytologic or pathologic diagnosis with PTC-related nuclear
changes, NRAS mutation, and male sex.

Conclusions: The prevalence of malignancy in patients with a preoperative diagnosis of follicular neoplasm was much higher in our study than in previous reports. Cytologic or pathologic PTC-related nuclear changes is a useful predictor of the presence of malignancy. Further studies must be conducted to support our results.

Keywords: Follicular neoplasm; atypia; thyroidectomy; thyroid carcinoma

Submitted May 15, 2020. Accepted for publication Sep 30, 2020.

doi: 10.21037 /gs-20-500

View this article at: http://dx.doi.org/10.21037/gs-20-500

\section{Introduction}

Thyroid nodules are one of the most common diseases in clinical practice. Worldwide, approximately $5 \%$ of women and $1 \%$ of men have palpable nodules (1-3). Recently, highresolution ultrasound (US) has been used for screening tests, and the diagnosis of thyroid nodules has greatly increased $(2,4)$. Thyroid nodules are very common, and most are benign, but approximately $5 \%$ to $10 \%$ are diagnosed as malignant (5).

Fine-needle aspiration cytology (FNAC) is the gold standard for diagnosing thyroid nodules with a simple procedure and high accuracy $(6,7)$. Although US-guided FNAC is significantly accurate in distinguishing between 
papillary thyroid carcinoma (PTC) and benign disease, it is difficult to distinguish follicular thyroid carcinoma (FTC) and follicular variant papillary thyroid carcinoma (fvPTC) from benign follicular adenoma $(8,9)$. FTC is distinguished from a follicular adenoma based on capsular invasion, extrathyroidal extension (ETE), vascular invasion, lymph node metastasis, or distant metastasis $(10,11)$.

Because of the risk of malignancy, with approximately $10 \%$ to $30 \%$ being follicular neoplasm or suspicious for follicular neoplasm $(12,13)$, diagnostic surgical resection is the long-established standard of management according to the American Thyroid Association (ATA) guidelines (14). This standard causes many patients to undergo unnecessary surgery of benign disease. In contrast, some patients who are diagnosed with FTC in surgical pathology should undergo completion thyroidectomy. Therefore, it is quite important to determine whether follicular neoplasm is benign or malignant before surgery.

Several studies have validated clinical or pathologic features for predicting malignancy in follicular neoplasms. Age, male sex, large nodule size, US features, and cytologic features have been reported as the main risk factors to define patients at risk for malignancy. However, there are still controversial predictors of malignancy in follicular neoplasms.

The objective of this retrospective study was to identify the clinical and cytologic/pathologic features to predict malignancy in patients preoperatively diagnosed with follicular neoplasm. We present the following article in accordance with STROBE reporting checklist (available at http://dx.doi.org/10.21037/gs-20-500).

\section{Methods}

\section{Patients}

In total, 425 patients preoperatively diagnosed with follicular neoplasm or suspicious for follicular neoplasm who underwent a thyroid operation, including total or less than total thyroidectomy, were retrospectively reviewed at Seoul St. Mary's Hospital (Seoul, Korea) from January 2010 to June 2018. Nine patients were excluded as they had inappropriate data. A total of 416 patients were analyzed by a complete review of medical charts and pathologic slide review. This study was conducted in accordance with the Declaration of Helsinki (as revised in 2013). This study was approved by the Institutional Review Board at Seoul St. Mary's Hospital, the Catholic University of Korea (IRB
No. KC19RESI0264) and the need for informed consent was waived due to the retrospective nature of this study.

\section{FNAC and core needle biopsy (CNB)}

All FNAC or CNB procedures were performed by US guidance by experienced radiologists. US-guided CNB was performed according to the following indications: (I) thyroid nodules with macrocalcifications, hypervascularity, or scanty aspirates by FNAC; (II) thyroid nodules with prior non-diagnostic/unsatisfactory FNAC; (III) thyroid nodules with suspicious features for malignancy on US but prior non-malignant FNAC. An 18-gauge, double-action, springactivated needle was used for CNB.

\section{Pathologic diagnosis using FNAC and CNB}

The diagnosis made using FNAC and CNB was based on the Bethesda system and included Bethesda category IV. The slides were examined by a single, blinded and experienced pathologist at our institution. Follicular neoplasms with more than $75 \%$ of Hürthle cell components were classified as Hürthle cell subtypes. Follicular neoplasms, other than the Hürthle cell subtype, were classified according to the type of atypia, namely, architectural (microfollicular formations, crowding, and isolated cells) or nuclear (nuclear enlargement, chromatin clearing, nuclear grooves, and nuclear membrane irregularity) (15). The nuclear atypia raised the concern for PTC, but was not diagnostic of malignancy. The specimen showing architectural atypia and nuclear atypia concerning for PTC was interpreted as follicular neoplasm with PTC-related nuclear changes. These findings were compared with the final surgical pathology.

\section{Statistical analysis}

Continuous variables were reported as the mean with standard deviation (SD), and categorical variables were provided as numbers with percentages. Student's $t$-test was used to compare continuous variables. Pearson's chisquare test or Fisher's exact test was performed to compare categorical variables between the two groups. Univariate and multivariate logistic regression analyses were performed to identify which factors were associated with the diagnosis of malignancy. Odds ratios (ORs) with $95 \%$ confidence intervals (CI) were calculated. A P value $<0.05$ was considered statistically significant. All statistical analyses 
Table 1 Baseline clinical characteristics of the study patients

\begin{tabular}{lc}
\hline Variable & $\mathrm{N}=416$ \\
\hline Age (years) & $47.1 \pm 13.7$ (range, 14-83) \\
Male:female & $1: 3.2$ \\
Male & $99(23.8)$ \\
Female & $317(76.2)$ \\
TSH (mlU/L) & $2.1 \pm 1.9$ (range, 0.01-25.3) \\
Method of diagnosis & $138(33.2)$ \\
FNAC & $278(66.8)$ \\
CNB & \\
Follicular neoplasm subtype & $337(81.0)$ \\
Conventional & $299(71.9)$ \\
Architectural atypia & $38(9.1)$ \\
PTC-related nuclear changes & $79(19.0)$ \\
Hürthle cell &
\end{tabular}

Data are expressed as patient's number (\%), or mean \pm SD. $\mathrm{TSH}$, thyroid stimulating hormone; FNAB, fine needle aspiration biopsy; CNB, core needle biopsy; PTC, papillary thyroid carcinoma.

were performed using IBM SPSS Statistics for Windows, version 23.0 (IBM Corp., Armonk, NY, USA).

\section{Results}

Baseline clinicopathological characteristics of the study patients

Table 1 presents the baseline clinical characteristics of the study patients who underwent thyroidectomy due to follicular neoplasm or suspicious for follicular neoplasm. The mean age of the study patients was $47.1 \pm 13.6$ years (range, 14-83 years), and 317 (76.2\%) patients were female. The mean TSH was $2.1 \pm 1.9 \mathrm{mIU} / \mathrm{L}$ (range, $0.01-$ $25.3 \mathrm{mIU} / \mathrm{L})$. In total, 278 (66.8\%) patients were diagnosed with thyroid nodules by CNB. There were 79 (19.0\%) patients diagnosed with the Hürthle cell subtype by FNAC or CNB. PTC-related nuclear changes and architectural atypia were diagnosed in $38(9.1 \%)$ and 299 (71.9\%) patients, respectively.

The baseline clinicopathological characteristics of the study patients are shown in Table 2. In total, 73 (17.5\%) patients underwent total thyroidectomy, and 343 (82.5\%) patients underwent lobectomy and/or contralateral
Table 2 Baseline clinicopathological characteristics of the study patients

\begin{tabular}{|c|c|}
\hline Variable & $\mathrm{N}=416$ \\
\hline \multicolumn{2}{|l|}{ Extent of operation } \\
\hline Less than total & $343(82.5)$ \\
\hline Total & $73(17.5)$ \\
\hline Malignancy/NIFTP & $209(50.2)$ \\
\hline Tumor size $(\mathrm{cm})$ & $2.4 \pm 1.6$ (range, $0.5-13.5)$ \\
\hline ETE & $10(6.8)$ \\
\hline NRAS & $49 / 220(22.3)$ \\
\hline $\mathrm{BRAF}^{\mathrm{V} 600 \mathrm{E}}$ & $14 / 193(7.3)$ \\
\hline Lymphatic invasion & $11 / 146(7.5)$ \\
\hline Vascular invasion & $31 / 146(21.2)$ \\
\hline Perineural invasion & $1 / 146(0.7)$ \\
\hline \multicolumn{2}{|l|}{ T category } \\
\hline $\mathrm{T} 1$ & $74(50.7)$ \\
\hline T2 & $51(34.9)$ \\
\hline T3 & $21(14.4)$ \\
\hline \multicolumn{2}{|l|}{$\mathrm{N}$ category } \\
\hline $\mathrm{N} 1 \mathrm{a}$ & $19(13.0)$ \\
\hline \multicolumn{2}{|l|}{ M category } \\
\hline M1 & $2(1.4)$ \\
\hline \multicolumn{2}{|l|}{ TNM stage } \\
\hline I & $135(92.5)$ \\
\hline II & $10(6.8)$ \\
\hline $\mathrm{IVb}$ & $1(0.7)$ \\
\hline
\end{tabular}

Data are expressed as patient's number (\%), or mean \pm SD. NIFTP, noninvasive follicular thyroid neoplasm with papillarylike nuclear features; ETE, extrathyroidal extension; T, tumor; $\mathrm{N}$, node; $M$, metastasis.

partial thyroidectomy. In total, 209 (50.2\%) patients were diagnosed with malignancy or noninvasive follicular thyroid neoplasm with papillary-like nuclear features (NIFTP). The mean tumor size was $2.4 \pm 1.6 \mathrm{~cm}$ (range, $0.5-13.5 \mathrm{~cm}$ ), and ETE was diagnosed in $10(6.8 \%)$ patients. The NRAS test was performed in 220 patients, of whom 49 (22.3\%) patients were positive. Likewise, the $B R A F^{V 600 E}$ test was performed in 193 patients, of whom 14 (7.3\%) patients were positive. In 146 patients diagnosed with malignancy, lymphatic, vascular and perineural invasion were diagnosed in 
Table 3 Surgical pathologic diagnosis of the study patients

\begin{tabular}{lc}
\hline Variable & $\mathrm{N}=416(\%)$ \\
\hline Non-malignant & $207(49.8)$ \\
Nodular hyperplasia & $47(11.3)$ \\
Follicular adenoma & $103(24.8)$ \\
Hürthle cell adenoma & $57(13.7)$ \\
NIFTP & $63(15.1)$ \\
Malignant & $146(35.1)$ \\
cPTC & $23(5.5)$ \\
fVPTC & $55(13.3)$ \\
FTC & $59(14.2)$ \\
HCC & $5(1.2)$ \\
Poorly DTC & $3(0.7)$ \\
MTC & $1(0.2)$ \\
\hline
\end{tabular}

NIFTP, noninvasive follicular thyroid neoplasm with papillarylike nuclear features; cPTC, conventional papillary thyroid carcinoma; fvPTC, follicular variant papillary thyroid carcinoma; FTC, follicular thyroid carcinoma; HCC, Hurthle cell carcinoma; DTC, differentiated thyroid carcinoma; MTC, medullary thyroid carcinoma.

$11(7.5 \%)$ patients, $31(21.2 \%)$ patients and $1(07 \%)$ patient, respectively. The numbers of patients with malignancy at each T stage were as follows: $74(50.7 \%)$ at stage 1, 51 (34.9\%) at stage 2, and $21(14.4 \%)$ at stage 3 . The numbers of patients diagnosed with $\mathrm{N} 1 \mathrm{a}$ and M1 were 19 (13.0\%) and $2(1.4 \%)$, respectively. The numbers of patients at each TNM stage were as follows: $135(92.5 \%)$ at stage I, 10 $(6.8 \%)$ at stage II, and $1(0.7 \%)$ at stage IVb.

\section{Surgical pathologic diagnosis of study patients}

The final surgical pathologic diagnosis of the study patients is provided in Table 3. Of these, 207 (49.8\%) patients were diagnosed with non-malignant disease, 47 $(11.3 \%)$ patients were diagnosed with nodular hyperplasia, $103(24.8 \%)$ patients were diagnosed with follicular adenoma, and $57(13.7 \%)$ patients were diagnosed with Hürthle cell adenoma. Sixty-three (15.1\%) patients were diagnosed with NIFTP. Of the 146 (35.1\%) patients diagnosed with malignant disease, FTC was the most diagnosed with $59(14.2 \%)$ patients, and fvPTC was the second most diagnosed with 55 (13.3\%) patients. The 49 NRAS mutation patients comprised 13 (26.5\%) fvPTCs and $2(4.1 \%)$ FTCs, and the 19 N1a patients comprised $10(52.6 \%)$ fvPTCs and 5 (26.3\%) FTCs. Of 38 patients with PTC-related nuclear changes, fvPTC was the most diagnosed with $16(42.1 \%)$ patients, and conventional PTC was the second most diagnosed with 7 (18.4\%) patients.

\section{Comparison of baseline clinicopathological characteristics between benign and malignant groups}

The results of the baseline clinicopathological characteristic comparisons between the benign and malignancy/NIFTP groups are shown in Table 4. There were no statistically significant differences in age, method of diagnosis and mean tumor size between the two groups. Malignancy/ NIFTP was diagnosed in $71(51.4 \%)$ and 138 (49.6\%) patients with FNAC and CNB, respectively, but there was no statistically significant difference between the groups $(\mathrm{P}=0.755)$. However, the proportion of male patients was significantly higher in the malignancy/NIFTP group than in the benign group $(\mathrm{P}=0.038)$. PTC-related nuclear changes was higher in the malignancy/NIFTP group than in the benign group $(\mathrm{P}<0.001)$. PTC-related nuclear changes was diagnosed significantly more frequently with CNB than with FNAC $(11.1 \%, 31 / 278$ vs. $5.1 \%, 7 / 138$; $\mathrm{P}=0.002$; not shown in table). ETE was significantly more frequent in the malignancy/NIFTP group than in the benign group $(\mathrm{P}<0.001)$, and the proportion of NRAS mutations and $B R A F^{V 600 E}$ mutation were significantly higher in the malignancy/NIFTP group $(\mathrm{P}=0.013$ and $\mathrm{P}<0.001$, respectively).

\section{Univariate and multivariate analyses of clinical parameters that influence the diagnosis of malignancy/ NIFTP}

Univariate and multivariate logistic regression analyses were performed to determine the independent risk factors associated with the diagnosis of malignancy/NIFTP (Table 5). Male sex was a significant risk factor for the diagnosis of malignancy (OR, 2.027; 95\% CI, 1.042 to 3.944; $\mathrm{P}=0.037)$. The NRAS mutation was confirmed as a significant risk factor in a multivariate analysis $(\mathrm{OR}, 2.483$; 95\% CI, 1.212 to 5.086; $\mathrm{P}=0.013)$. Among the various risk factors, PTC-related nuclear changes was identified as the most significant risk factor for the diagnosis of malignancy/ NIFTP in both univariate and multivariate analyses $(\mathrm{OR}$, 10.762; $95 \%$ CI, 3.002 to 38.575 ; $\mathrm{P}<0.001)$. 
Table 4 Comparison of baseline clinicopathological characteristics between benign and malignancy groups

\begin{tabular}{|c|c|c|c|}
\hline Variable & Benign $(n=207)$ & Malignancy/NIFTP $(n=209)$ & $P$ value \\
\hline Male & $40(19.7 \%)$ & $59(28.2 \%)$ & 0.038 \\
\hline Method of diagnosis & & & 0.755 \\
\hline FNAC & $67(48.6 \%)$ & $71(51.4 \%)$ & \\
\hline Follicular neoplasm subtype & & & $<0.001$ \\
\hline Architectural atypia & $149(72.0 \%)$ & $150(71.8 \%)$ & \\
\hline PTC-related nuclear changes & $4(1.9 \%)$ & $34^{\dagger}(16.3 \%)$ & \\
\hline Hürthle cell & $54(26.1 \%)$ & $25(11.9 \%)$ & \\
\hline NRAS positive & 15/108 (13.8\%) & $34 / 112(30.4 \%)$ & 0.013 \\
\hline $\mathrm{BRAF}^{\mathrm{V} 600 \mathrm{E}}$ positive & 0/91 (0\%) & $14 / 102(13.7 \%)$ & $<0.001$ \\
\hline
\end{tabular}

Data are expressed as patient's number (\%), or mean \pm SD. A statistically significant difference was defined as $\mathrm{P}<0.05$. ${ }^{\dagger}$, the 34 PTCrelated nuclear changes patients of malignant group comprised 7 (20.6\%) cPTCs, 23 (67.6\%) fvPTC and 4 (11.8\%) FTCs. NIFTP, noninvasive follicular thyroid neoplasm with papillary-like nuclear features; FNAB, fine needle aspiration biopsy; CNB, core needle biopsy, ETE, extrathyroidal extension; cPTC, conventional papillary thyroid carcinoma; fvPTC, follicular variant papillary thyroid carcinoma; FTC, follicular thyroid carcinoma.

\section{Discussion}

Preoperative diagnosis of malignancy for follicular neoplasm remains difficult. US-guided FNAC is the most commonly used safe method for evaluating thyroid nodules with high sensitivity and specificity, but it has limited accuracy with follicular lesions. In spite of various studies, the distinction between benign and malignant disease from follicular neoplasm is still difficult on cytologic testing. Therefore, surgery is recommended for the diagnosis and treatment of follicular neoplasms.

Various methods, such as pathologic testing by CNB, US features, and NRAS testing, have been investigated to differentiate benign and malignant disease from follicular neoplasm. Min et al. reported that preoperative CNB had no advantage over FNAC in predicting malignancy (16). Lee $e t$ al. also reported that follicular neoplasm was frequently diagnosed in $\mathrm{CNB}$, but the rates of malignancy were equivalent to FNAC (17). In the present study, CNB was performed in $278(66.8 \%)$ patients, but there was no significant difference from FNAC in the diagnostic rate of malignancy/NIFTP ( $51.4 \%$ vs. $48.6 \%, \mathrm{P}=0.755$ ). Another group reported that US features, such as solid echo structure, microcalcification, or hypoechoic pattern, were associated with malignancy (18), but US has the disadvantage of being a subjective method used by radiologists.

Bongiovanni et al. reported that the malignancy rate of follicular neoplasm was $26.1 \%$ (19), but 146 (35.1\%) patients were diagnosed with malignancy in the final surgical pathologic results in this study. Our malignancy rate was higher than that of previous reports. The first reason for these different results is the difference in ethnicity. Previous studies have been conducted mostly in the United States, but Korea is one of the countries with the highest prevalence of thyroid carcinoma. Yim et al. reported that the malignancy rate is $48 \%$ in Korea (20). The second reason is that Korea is an iodine-sufficient region. Iodine deficiency is associated with FTC, but iodine sufficiency is associated with PTC. Of the malignant cases, PTC was the most frequently diagnosed (53.4\%, 78/146), and FTC was the second most frequently diagnosed (40.4\%, $59 / 146$ ) in this present study. In addition, almost one third of the malignancy $(37.7 \%, 55 / 146)$ was fvPTC, which could signify that we have misdiagnosed or underdiagnosed a significant number of fvPTCs as follicular neoplasms. 
Table 5 Univariate and multivariate analyses of clinical parameters which influence on the diagnosis of malignancy/NIFTP

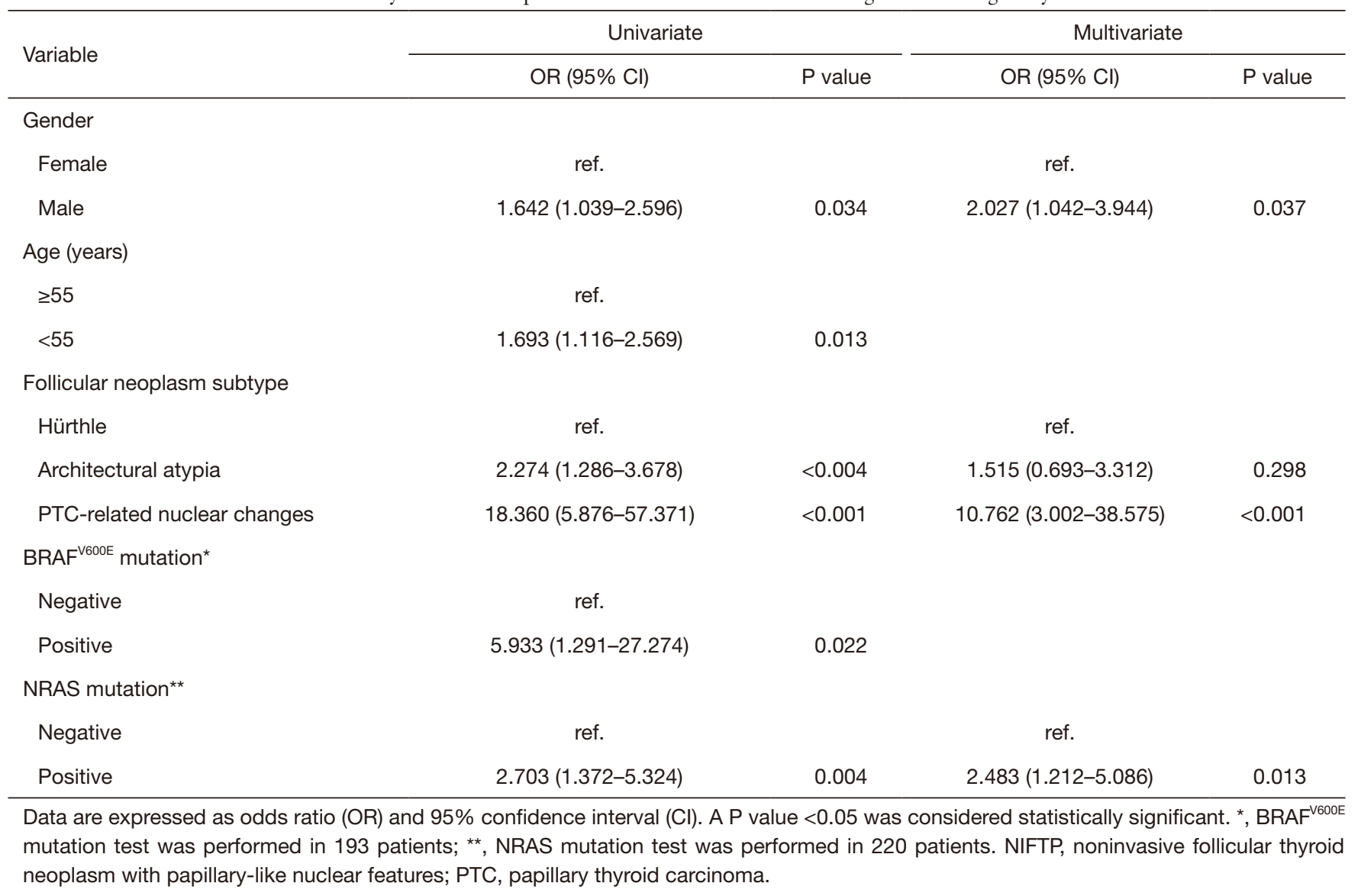

We investigated the clinical, cytologic or pathologic features of 416 patients with preoperatively diagnosed follicular neoplasms or suspicious for follicular neoplasms to identify which features influence the diagnosis of malignancy. The results showed that three different factors, namely, male sex, PTC-related nuclear changes and NRAS mutation, are predictors of malignancy.

A cytologic or pathologic pattern of architectural atypia was associated with follicular neoplasm (21). The architectural atypia was not significantly different between the benign and malignancy/NIFTP groups $(72 \%$ vs. $71.8 \%)$. In contrast, cytologic or pathologic PTC-related nuclear changes was more common in malignancy/NIFTP (1.9\% vs. $16.3 \%)$ and was identified as the most powerful predictor of malignancy/NIFTP in the multivariate analysis (OR 10.762, $\mathrm{P}<0.001$ ). This finding emphasizes that PTCrelated nuclear changes is more important than architectural atypia for predicting malignancy/NIFTP in patients with follicular neoplasms.
Several studies have examined the factors that help predict malignancy in preoperatively diagnosed follicular neoplasm. The first study analyzed 368 surgical thyroid specimens. The authors found that $60 \%$ of nodules with cytologic PTC-related nuclear changes were malignant. They concluded that cytologic PTC-related nuclear changes consistent with a follicular neoplasm conferred a high risk of malignancy (22). The other study investigated 98 follicular neoplasms. It showed that the malignant proportions of follicular neoplasms with atypia and without atypia were $44.4 \%$ and $6.8 \%$, respectively. They reported that follicular neoplasms with atypia were predictive of malignancy, as well (9).

Among 38 patients with PTC-related nuclear changes, $16(42.1 \%)$ patients were diagnosed with fvPTC. This result suggests that there might be a significant relative risk of fvPTC associated with cytologic or pathologic PTC-related nuclear changes over other types of thyroid carcinomas, such as FTC and conventional PTC. Several studies were 
consistent with our results $(23,24)$, but further work is required to establish the durability of the relationship between PTC-related nuclear changes and fvPTC.

Clinical variables have been investigated to predict malignancy from follicular neoplasms. Several studies demonstrated that male sex was significantly associated with a diagnosis of malignancy $(25,26)$. The present study also showed that male sex was predictive of malignancy/NIFTP in the multivariate analysis (OR: 2.027, $\mathrm{P}=0.037$ ).

Tuttle et al. reported that a tumor size greater than $4 \mathrm{~cm}$ was associated with the risk of malignancy (27). Schlinkert et al. also found that the risk of malignancy in follicular neoplasm was higher in larger tumors (28). Another study showed that a tumor size greater than $2.1 \mathrm{~cm}$ increased the risk of malignancy (23). However, our results showed no significant difference between the benign and malignant groups with tumor size.

Recently, molecular biomarkers, such as $B R A F$, RAS, RET-PTC, or PAX8-PPARr mutations, have been investigated for the diagnosis of thyroid nodules. The $B R A F$ mutation has been observed in up to $83 \%$ of PTC (29-31), whereas the NRAS mutation has been commonly observed in follicular neoplasms (32). In the current study, the NRAS mutation was observed in $49(22.3 \%)$ of 220 patients, and there was a statistically significant difference between the benign and malignancy/NIFTP groups ( $13.8 \%$ vs. $30.4 \%, \mathrm{P}=0.013)$. In the multivariate analysis, the NRAS mutation was identified as an independent risk factor for the diagnosis of malignancy/NIFTP, as well (OR: 2.483, $\mathrm{P}=0.013$ ). Bae et al. reported that the overall malignancy rate was significantly higher in the NRAS mutation than with no mutation (33). Preoperative NRAS tests using FNAC or CNB specimens may help improve the prediction of malignancy in patients with follicular neoplasm.

This study has several limitations. First, this study was designed as a retrospective study in nature. In addition, there may have been selection bias, as all the patients were enrolled from a single tertiary institution, and they do not reflect the entire patient population. Third, the NRAS test was not performed in all patients and was performed using a surgical specimen. Finally, we conducted this study with a relatively small sample size. This limitation could be overcome by conducting a multicenter study in the future. The most important strength of this study is that because all the cytologic and pathologic slides were reviewed by a single, experienced pathologist, so there is uniformity of diagnosis.

\section{Conclusions}

Based on the results of our study, the prevalence of malignancy in patients with a preoperative diagnosis of follicular neoplasm was much higher than in previous reports. Cytologic or pathologic PTC-related nuclear changes is a useful predictor for the presence of malignancy. Further studies must be conducted to support our results.

\section{Acknowledgments}

This study was presented at the $89^{\text {th }}$ Annual Meeting of the American Thyroid Association.

Funding: This research was supported by Basic Science Research Program through the National Research Foundation of Korea (NRF) funded by the Ministry of Education (NRF-2015R1D1A1A01058798).

\section{Footnote}

Reporting Checklist: The authors have completed the STROBE reporting checklist. Available at http://dx.doi. org/10.21037/gs-20-500

Data Sharing Statement: Available at http://dx.doi. org/10.21037/gs-20-500

Peer Review File: Available at http://dx.doi.org/10.21037/gs20-500

Conflicts of Interest: All authors have completed the ICMJE uniform disclosure form (available at http://dx.doi. org/10.21037/gs-20-500). CKJ serves as an unpaid editorial board member of Gland Surgery from July 2019 to June 2021. The other authors have no conflicts of interest to declare.

Etbical Statement: The authors are accountable for all aspects of the work in ensuring that questions related to the accuracy or integrity of any part of the work are appropriately investigated and resolved. The study was conducted in accordance with the Declaration of Helsinki (as revised in 2013). The study was approved by Ethics Committee of the Institutional Review Board at Seoul St. Mary's Hospital, the Catholic University of Korea (IRB No.: KC19RESI0264) and individual consent for this retrospective analysis was waived. 
Open Access Statement: This is an Open Access article distributed in accordance with the Creative Commons Attribution-NonCommercial-NoDerivs 4.0 International License (CC BY-NC-ND 4.0), which permits the noncommercial replication and distribution of the article with the strict proviso that no changes or edits are made and the original work is properly cited (including links to both the formal publication through the relevant DOI and the license). See: https://creativecommons.org/licenses/by-nc-nd/4.0/.

\section{References}

1. Davies L, Welch HG. Increasing incidence of thyroid cancer in the United States, 1973-2002. JAMA 2006;295:2164-7.

2. Lin JD, Chao TC, Huang BY, et al. Thyroid cancer in the thyroid nodules evaluated by ultrasonography and fineneedle aspiration cytology. Thyroid 2005;15:708-17.

3. Wang C, Crapo LM. The epidemiology of thyroid disease and implications for screening. Endocrinol Metab Clin North Am 1997;26:189-218.

4. Wiest PW, Hartshorne MF, Inskip PD, et al. Thyroid palpation versus high-resolution thyroid ultrasonography in the detection of nodules. J Ultrasound Med 1998;17:487-96.

5. Hegedüs L. The thyroid nodule. N Engl J Med 2004;351:1764-71.

6. Su DH, Liao KM, Hsiao YL, et al. Determining when to operate on patients with Hashimoto's thyroiditis with nodular lesions: the role of ultrasound-guided fine needle aspiration cytology. Acta Cytol 2004;48:622-9.

7. Sclabas GM, Staerkel GA, Shapiro SE, et al. Fineneedle aspiration of the thyroid and correlation with histopathology in a contemporary series of 240 patients. Am J Surg 2003;186:702-9; discussion 709-10.

8. Mikosch P, Gallowitsch H, Kresnik E, et al. Value of ultrasound-guided fine-needle aspiration biopsy of thyroid nodules in an endemic goitre area. Eur J Nucl Med 2000;27:62-9.

9. Goldstein RE, Netterville JL, Burkey B, et al. Implications of follicular neoplasms, atypia, and lesions suspicious for malignancy diagnosed by fine-needle aspiration of thyroid nodules. Ann Surg 2002;235:656.

10. DeMay RM. Follicular lesions of the thyroid: W(h)ither follicular carcinoma? Am J Clin Pathol 2000;114:681-3.

11. LiVolsi VA, Baloch ZW. Follicular-patterned tumors of the thyroid: the battle of benign vs. malignant vs. so-called uncertain. Endocr Pathol 2011;22:184-9.
12. Yang J, Schnadig V, Logrono R, et al. Fine-needle aspiration of thyroid nodules: a study of 4703 patients with histologic and clinical correlations. Cancer 2007;111:306-15.

13. Baloch ZW, Cibas ES, Clark DP, et al. The National Cancer Institute Thyroid fine needle aspiration state of the science conference: a summation. Cytojournal 2008;5:6.

14. Haugen BR, Alexander EK, Bible KC, et al. 2015 American Thyroid Association management guidelines for adult patients with thyroid nodules and differentiated thyroid cancer: the American Thyroid Association guidelines task force on thyroid nodules and differentiated thyroid cancer. Thyroid 2016;26:1-133.

15. Gan TRX, Nga ME, Lum JHY, et al. Thyroid cytologynuclear versus architectural atypia within the "Atypia of undetermined significance/follicular lesion of undetermined significance" B ethesda category have significantly different rates of malignancy. Cancer Cytopathol 2017;125:245-56.

16. Min HS, Kim JH, Ryoo I, et al. The role of core needle biopsy in the preoperative diagnosis of follicular neoplasm of the thyroid. Apmis 2014;122:993-1000.

17. Lee SH, Park GS, Jung SL, et al. Core-needle biopsy for the preoperative diagnosis of follicular neoplasm in thyroid nodule screening: a validation study. Pathol-Research Prac 2016;212:44-50.

18. Gulcelik NE, Gulcelik MA, Kuru B. Risk of malignancy in patients with follicular neoplasm: predictive value of clinical and ultrasonographic features. Arch Otolaryngol Head Neck Surg 2008;134:1312-5.

19. Bongiovanni M, Spitale A, Faquin WC, et al. The Bethesda system for reporting thyroid cytopathology: a meta-analysis. Acta Cytol 2012;56:333-9.

20. Yim JH, Kim EY, Kim WG, et al. Postoperative findings of the cytological diagnosis of follicular neoplasm or Hürthle cell neoplasm and the risk of malignancy. Endocrinol Metabol 2010;25:316-20.

21. Carpi A, Nicolini A, Sagripanti A, et al. Large-needle aspiration biopsy for the preoperative selection of palpable thyroid nodules diagnosed by fine-needle aspiration as a microfollicular nodule or suspected cancer. Am J Clin Pathol 2000;113:872-7.

22. Kelman AS, Rathan A, Leibowitz J, et al. Thyroid cytology and the risk of malignancy in thyroid nodules: importance of nuclear atypia in indeterminate specimens. Thyroid 2001;11:271-7.

23. Yang GC, Goldberg JD, Ye PX. Risk of malignancy in follicular neoplasms without nuclear atypia: statistical 
analysis of 397 thyroidectomies. Endocr Pract 2003;9:510-6.

24. Ustun B, Chhieng D, Prasad ML, et al. Follicular variant of papillary thyroid carcinoma: accuracy of FNA diagnosis and implications for patient management. Endocr Pathol 2014;25:257-64.

25. Baloch ZW, Fleisher S, LiVolsi VA, et al. Diagnosis of "follicular neoplasm": a gray zone in thyroid fine-needle aspiration cytology. Diagn Cytopathol 2002;26:41-4.

26. Davis NL, Gordon M, Germann E, et al. Clinical parameters predictive of malignancy of thyroid follicular neoplasms. Am J Surg 1991;161:567-9.

27. Tuttle RM, Lemar H, Burch HB. Clinical features associated with an increased risk of thyroid malignancy in patients with follicular neoplasia by fine-needle aspiration. Thyroid 1998;8:377-83.

28. Schlinkert RT, Van Heerden JA, Goellner JR, et al. editors. Factors that predict malignant thyroid lesions when fine-

Cite this article as: Kim K, Jung CK, Lim DJ, Bae JS, Kim JS. Clinical and pathologic features for predicting malignancy in thyroid follicular neoplasms. Gland Surg 2021;10(1):50-58. doi: 10.21037 /gs-20-500 needle aspiration is "suspicious for follicular neoplasm". Mayo Clin Proc: Elsevier, 1997.

29. Kim TY, Kim WB, Song JY, et al. The BRAFV600E mutation is not associated with poor prognostic factors in Korean patients with conventional papillary thyroid microcarcinoma. Clin Endocr 2005;63:588-93.

30. Fukushima T, Suzuki S, Mashiko M, et al. BRAF mutations in papillary carcinomas of the thyroid. Oncogene 2003;22:6455.

31. Cho U, Oh WJ, Bae JS, et al. Clinicopathological features of rare BRAF mutations in Korean thyroid cancer patients. J Korean Med Sci 2014;29:1054-60.

32. Liu RT, Hou CY, You HL, et al. Selective occurrence of ras mutations in benign and malignant thyroid follicular neoplasms in Taiwan. Thyroid 2004;14:616-21.

33. Bae JS, Choi SK, Jeon S, et al. Impact of NRAS mutations on the diagnosis of follicular neoplasm of the thyroid. Int J Endocrinol 2014;2014:289834. 